\title{
Index nominum ad Vol. 159
}

Angelov, A., 54 Arnold, M., 335 (S) Auclaire, M., 250 (B) Berge, B. S. ten, 359 Bergemann, E., 262 (S) Berger, H., 250 (B) Berger, M., 277 (S) Bickenbach, W., 254 (B) Bigler, R., 266 (S)

Bompiani, A., 256 (B) Brunschwig, A., 189 (B) Butler, R., 258 (S) Caldeyro-Barcia, R., 254 (B) Cernoch, A., 377 Cloeren, S., 3 Constam, G. R., 193 (S) Cordey, R., 393 Debiasi, E., 98 400

Index nominum ad Vol. 159

Deucher, F., 187 (B)

Diepgen, P., 128 (B)

Döring, G. K., 254 (B)

Erb, H., 25, 309 (S)

Fassolt, A., 286 (S)

Flury, R., 266 (S)

Gasparri, F., 159

Gloor, R. D. 262 (S)

Graf, K., 384

Gray, A. B., 253 (B)

Greep, R. 0. 256 (B)

Hauser, G. A., 167, 255 (B), 286 (S)

Heller, H., 254 (B)

Högler, H., 253 (B)

Hottinger, A., 250 (B)

Hürlimann, F., 167

Ingiulla, W., 159

Ivanov, I., 54

Jirásek, J. E., 377

Jung, G., 396 (B)

Jung, H., 251 (B)

Kanka, J., 129

Kern, G., 254 (B)

Kietlińska, Z, 47

Klotz, B., 189 (B)

Roller, T., 307 (S)

König, P. A., 107, 396 (B)

Kräubig, H., 185

Laman, A., 253 (B)

Lauritzen, C, 365

Lehmann, W.-D., 365

Louros, N. C, 251 (B) 
Ludwig, K. S., 309 (S)

Macku, F., 129

Mall, M., 307 (S)

Mall-Häfeli, M., 3

Malz, S., 351

Marsiková, L., 129

Meylan, J., 65

Miller, G., 187 (B)

Moser, H., 258 (S)

Müller, C, 190 (B)

Müller, D., 143

Müller, J. H., 321 (S)

Muralt, G., von, 258 (S)

Muth-Engelhardt, 191 (B)

Narik, G., 177

Obolensky, W., 329 (S)

Papadia, S., 98

Perrault, M., 189 (B)

Polvani, F., 256 (B)

Rabinowitsch, W., 153

Rakilovska, I., 54

Rehsteiner, H. P., 316 (S)

Reisenauer, R., 129

Renggli, I., 374

Richter, R. H. H., 335 (S)

Richterich, R., 252 (B)

Rippmann, E. T., 33

Roszkowski, I., 47

Rust, T., 215 (S)

Sang Kook Kim, 341

Sauter, H., 296 (S)

Schätzing, E., 190 (B)

Schneider, J., 341

Schreiner, W. E., 290 (S), 396 (B)

Schroder, R., 188 (B)

Schubiger, V., 286 (S)

Semm, K., 61

Siebenmann, R., 266 (S)

Soiva, K, 125

Stamm, 0., 266 (S)

Stoddard, J. F., 64 (B)

Storr, A., 251 (B)

Stucki, D., 190 (B)

Uher, J., 377

Villee, C. A., 290 (S)

Vladov, E., 54 
Widlocher, D., 189 (B)

Willi, H., 234 (S)

Wolff, E. de, 251 (B)

W. Wyss, H. J., 301 (S)

Zeilinger, I., 25 